Culture et histoire dans l'espace roman

$4 \mid 2010$

Les représentations du corps dans la litterature latinoaméricaine

\title{
La chute des corps selon Fernando Vallejo : El Desbarrancadero
}

\section{Thomas Barège}

\section{OpenEdition}

\section{Journals}

Édition électronique

URL : https://journals.openedition.org/cher/8485

DOI : $10.4000 /$ cher.8485

ISSN : 2803-5992

Éditeur

Presses universitaires de Strasbourg

Édition imprimée

Date de publication : 30 juin 2010

Pagination : 129-139

ISBN : 978-2-35410-007-0

ISSN : 1968-035X

Référence électronique

Thomas Barège, «La chute des corps selon Fernando Vallejo : El Desbarrancadero », reCHERches [En ligne], 4 | 2010, mis en ligne le 15 décembre 2021, consulté le 27 janvier 2022. URL : http:// journals.openedition.org/cher/8485; DOI : https://doi.org/10.4000/cher.8485

\section{(c) (i) (ㅇ)}

Ce(tte) œuvre est mise à disposition selon les termes de la Licence Creative Commons Attribution -

Pas d'Utilisation Commerciale - Partage dans les Mêmes Conditions 4.0 International. 


\title{
La chute des corps selon Fernando Vallejo: El Desbarrancadero
}

\author{
THOMAS BARÈGE \\ Université d'Orléans
}

Cernando Vallejo, dans son récit ${ }^{1}$ El Desbarrancadero (publié en 2001,
récompensé par le prix Rómulo Gallegos en 2003) met en scène le retour d'un narrateur - qui, comme d'habitude chez Vallejo, ressemble beaucoup à l'auteur - dans la maison de son enfance pour venir en aide à son frère en train de mourir du sida. Ce retour enclenche un processus mémoriel et amène à une sorte de superposition dans le récit entre l'agonie du frère et celle du père, résultant d'un cancer et survenue quelques années auparavant.

Fidèle à son écriture, Fernando Vallejo, continue, dans ce récit, ses imprécations contre son pays, la Colombie, contre l'Église, le pouvoir, mais aussi contre Dieu, clé et origine de tous les problèmes. Comme dans $\mathrm{La}$ Virgen de los sicarios, la violence est omniprésente et meurtrit les corps, à ceci près qu'ici, ce n'est plus tant la violence qui est au centre du roman, mais bien le corps lui-même, dans sa faiblesse (presque tous les personnages sont plus ou moins diminués physiquement) voire dans son délitement. Le corps malade est l'image obsédante du récit. Dans une époque qui voit surtout

1 Peut-être devrait-on plutôt parler d'autofictions pour une grande partie des ouvrages de F. Vallejo, puisqu'ils en ont tous les caractères. Dans le cas présent, l'ambiguïté est d'autant plus grande que la photo de couverture de El desbarrancadero est une photo personnelle de l'auteur, mais lorsque le narrateur décrit et commente (p. 160) l'une des siennes, on reconnaît aisément la photo de couverture. À propos de la dimension autobiographique de l'œuvre de $\mathrm{F}$. Vallejo, on pourra se référer à l'analyse du cycle El río del tiempo (Semilla Durán, 2004). 
dans le corps un motif érotique, Vallejo se singularise en plaçant le corps beaucoup plus du côté de Thanatos que d'Éros. Nous ferons à plusieurs reprises référence à $L a$ Virgen de los sicarios car une véritable continuité est lisible entre les deux récits.

En nous intéressant tout d'abord aux passages où le corps est montré dans toute sa faiblesse, nous chercherons à montrer à quel point le corps est peu érotique. C'est le registre scientifique qui est majoritairement convoqué pour décrire le corps, et ce, au travers de plusieurs disciplines. On remarque alors que Vallejo s'inscrit dans la continuité de certains discours traditionnels tout en les réactualisant. Au final, El desbarrancadero nous apparaît comme un memento mori iconoclaste, revu et corrigé par la science moderne: plus question d'espérer l'autre monde, ou une rédemption après le pourrissement. Je souhaiterais ainsi montrer comment Vallejo développe une vision du corps peut-être moins originale qu'à contretemps, en faisant de ce corps une sorte de carrefour, entre les époques et les disciplines.

La vision contemporaine du corps est souvent érotique, et de la part d'un auteur homosexuel, on attendrait presque une célébration du corps masculin conforme à certains clichés en cours à propos de la culture gay. Le moins que l'on puisse dire est que F. Vallejo prend le contre-pied de cette attente. Dès La Virgen de los sicarios, l'auteur avait mis en œuvre une sorte de stratégie d'évitement de l'érotique: "Les evito toda descripción pornográfica y sigamos» (Vallejo 2006: 11) peut-on lire lors de la première scène d'amour entre le narrateur et son jeune ami. Cette stratégie n'est pas dénuée d'humour lorsque quelques pages plus loin, le narrateur s'adresse de la manière suivante à son lecteur:

Nuestras noches encendidas de pasión, yo abrazado a mi ángel de la guarda y él a mí con el amor que me tuvo, porque debo consignar aquí, sin jactancias ni presunción, lo mucho que me quería. Es de poca caridad, ya sé, exhibir la dicha propia ante la desgracia ajena, contarle historias de amor libre a quien vive prisionero, encerrado, casado, con mujer gorda y propia y cinco hijos comiendo, jodiendo y viendo televisión. Mas dejemos el aparato y sigamos, exhibiendo plata ante el mendigo. (Vallejo 2006: 24)

La description en elle-même des corps ou des rapports reste très pudique, mais la suggestion, sur le mode de la moquerie, permet la présence d'un imaginaire érotique. L'argument de la charité resurgit lors de la rencontre du deuxième tueur, développé dans son aspect blasphématoire, puisqu'à la charité s'ajoute l'image de la Vierge mêlée à la nudité érotique: 
Vuelvo y repito: no hay que contar plata delante del pobre. Por eso no les pienso contar lo que esa noche antes de dormir pasó. Básteles saber dos cosas: Que su desnuda belleza se realzaba por el escapulario de la Virgen que le colgaba en el pecho. Y que al desvestirse se le cayó un revólver. (Vallejo 2006: 99)

C'est donc uniquement par «charité chrétienne» que le narrateur passe sous silence les détails de ses nuits érotiques avec ses jeunes amants. La tonalité est donc plutôt riante; ce n'est plus du tout le cas dans El desbarrancadero. Le corps érotique n'est plus présent que sur le mode du souvenir: souvenirs que le narrateur et son frère Darío évoquent avec une sorte de nostalgie. La beauté érotique appartient au passé: «Muchachitos y muchachos de Junín, idos sois. Os borró de un plumazo Cronos, el descabezador de bellezas. Y hoy por mi pobre calle transitan zombies y saltapatrases, que es en lo que se ha convertido esta raza asesina» (Vallejo 2001: 107). L'expression «raza asesina» évoque clairement les personnages centraux du récit de 1994. En effet les «sicarios» sont pratiquement absents de El desbarrancadero. Cette transformation est tout à fait exemplaire du second récit: c'est le mortvivant qui a pris la place de l'éphèbe. Les meurtriers ont laissé la place aux mourants. Le passé lui-même est en sursis puisque la mémoire de Darío est fautive: il ne se souvient plus du plus beau des garçons avec lequel il a eu une aventure (Vallejo 2001 : 162).

En premier lieu des mourants, apparaît bien sûr le frère du narrateur, Darío, en phase terminale du sida. Resurgit à l'occasion de cette agonie, le père du narrateur, mort quelques années auparavant d'un cancer, et dont la mort refait surface dans l'esprit du narrateur. On aurait pu attendre, du fait du mode de transmission du sida, que cette maladie serve de lien entre le corps érotique et le corps mourant. En réalité, le récit en est à l'étape suivante, la contamination n'est plus d'actualité, c'est la mort qui focalise toute l'attention du narrateur.

Même s'il est omniprésent, nous y reviendrons, le sida n'est pas le seul responsable: les corps sont par nature, dirions-nous, diminués. Le professeur de musique du narrateur, Don Roberto est «sordo como Beethoven» (Vallejo 2001: 27) et il n'est pas le seul, la mère du narrateur est elle aussi sourde au point de détruire les tympans des autres (Vallejo 2001: 83), de plus elle n'a pas toute sa santé mentale puisqu'elle est constamment appelée la Loca; le narrateur lui-même a des problèmes de dents suite à un accident (Vallejo 2001: 72). Lors de l'agonie du père l'une des femmes de la famille est décrite, quant à elle, de la manière suivante: « estaba la pobre tan flaca que se 
le podía tomar una radiografía con una vela. Y así era en efecto, la angustia la iba a matar» (Vallejo 2001: 118). Un autre des frères du narrateur se suicide en sautant par une fenêtre: c'est donc le portrait d'une famille exsangue que dresse le narrateur. Je passerai rapidement sur les conséquences multiples de la maladie du frère Darío ${ }^{2}$, aisément imaginables et fréquemment évoquées au cours du récit.

En revanche, il y a un aspect du discours concernant le sida qui est beaucoup plus original: discours métaphorique ou pseudo-métaphorique, appelons-le ainsi faute de mieux pour le moment. Le sida n'est alors pas le comparé mais le comparant. Le sida devient une sorte de référent métaphorique de la dévastation: la mère du narrateur, la Loca, dont le portrait tout au long du récit est terrible, est décrite ainsi: «era más dañina que un sida» (Vallejo 2001: 69). Ailleurs, sida et Internet sont mis sur le même plan des fléaux: «estas malditas plagas del sida y del Internet» (145). Poussant le paradigme jusqu'au bout, le sida est conçu comme le concurrent de la vieillesse, métaphore de la mort et de la déchéance. Et comme la vie a pour fin la mort, c'est la vie elle-même qui est l'équivalent du sida. La première occurrence de cette thématique apparaitt lors d'une exhortation au carpe diem:

¿Va a dejar uno de vivir por cuidar un sida? La vida es un sida. Si no miren a los viejos: débiles, enclenques, inmunosuprimidos, con manchas por todo el cuerpo y pelos en las orejas que les crecen y les crecen mientras se les encoje el pipí. Si eso no es sida entonces yo no sé qué es. (48)

Assez rapidement, l'expression va évoluer vers une formulation syntaxique plus cinglante encore: «el sida de la vejez»(52) puis «el sida de esta vida» (139). Le frère du narrateur qui s'est suicidé à 25 ans, l'a fait parce qu'il était «enfermo de lucidez» (97), c'est-à-dire ne sachant que trop bien que la vie est sans espoir. Tout cela participe donc d'un pessimisme assez peu nuancé. Le sida sert de référent absolu dans le néfaste et cela mène assez loin: Internet, disions-nous, lui est comparé. En soi cette comparaison n'est pas particulièrement intéressante, elle ne fait qu'alimenter la tonalité réactionnaire des écrits de Vallejo, mais elle se charge de sens si on la met en parallèle avec un autre passage:

Que le habían mandado [a Darío] sus amigos de Bogotá, cuando se enteraron de que estaba tan enfermo, un compact disc por el Internet o sideroespacio.

2 Diarrhée $(24,68)$, perte de poids $(24,41,177)$, perte de mémoire (162), taches dues au sarcome de Kaposi (168), inflammation des rétines (177)... 
¿Un compact disc? O yo no estaba enterado de los últimos adelantos de la ciencia, o el sida le estaba perturbando a Darío el juicio. (67).

Passons sur le fait que le narrateur ne soit pas très au fait des possibilités qu'offre Internet. Internet est assimilé au «sideroespacio», et lorsque l'on sait qu'Internet est aussi assimilé au fléau du sida, par un jeu de mot, «sideroespacio» prend un autre sens, celui d'espace sidéen et non plus celui d'espace sidéral. Comme si la maladie venait à contaminer le monde entier, le texte que nous lisons évidemment, mais aussi la langue elle-même.

Pour aller plus loin encore, à la fois dans le pessimisme et dans l'idée de métaphoriser la maladie, il faut s'intéresser à un passage où le narrateur parle de la syphilis qu'a également contractée son frère; la maladie de «tanta prosapia » est décrite comme une danseuse au charme sulfureux, experte dans la danse des sept voiles (45). Cette métaphorisation, cette personnification de la maladie, passe une fois de plus par le registre du corps étant donné que la danse est par excellence l'art du corps. Il semble donc que Vallejo veuille nous signifier que le corps est indéfectiblement et uniquement lié à la maladie, que les deux sont inséparables.

En parallèle à cette personnification de la maladie, le lecteur constate le phénomène strictement inverse: une certaine déshumanisation du corps tout au long du récit. Celle-ci se manifeste à différents degrés : animalisation, lorsque le narrateur donne à son frère un traitement contre la diarrhée qui est en réalité pour les bovins parce que «no es tanta la diferencia entre la humanidad y los bovinos como no sea que las mujeres producen con dos tetas menos leche que las vacas con cinco o seis» (15). Animalisation toujours lorsque la mère est comparée à un chien: "Como un perro orina para indicar que ahí pasó, la Loca se pasó la vida pariendo hijos » (195). Mais l'on peut descendre encore dans l'échelle de l'être.

La réification est présente dans au moins deux exemples du début du récit. Le narrateur constate que son frère a fini le savon: "Jabón no había. Que se acabó. También se acabó. Todo en esta vida se acaba. Y ahora el que estaba acabando era él [Darío], sin que ni Dios ni nadie pudiera evitarlo» (12). Le parallèle entre le savon qui est fini et le frère en train de mourir au travers de la répétition du verbe "acabar», assez impropre dans le second cas, est vraiment dégradant pour le corps. Mourant, le corps n'est déjà plus un être vivant, mais simplement une matière parmi d'autres. Comme la dimension médicale est omniprésente dans le récit, le terme de «caso» (en particulier «caso perdido», 11) apparaît lui aussi, ce qui enlève toute individualité humaine au personnage: le corps est, pour les médecins (à propos desquels 
nous allons revenir par la suite), mais aussi pour le narrateur, un simple objet d'étude, et ce, dans tous les sens du terme «objet».

Tout un paradigme métaphorique vient renforcer cette idée selon laquelle le corps n'est qu'un objet, à peine différent des autres: il s'agit de l'isotopie de la mécanique. Le lien s'établit dans le texte au travers de comparaisons ou de métaphores, surtout présentes dans la première moitié du récit. Une fois seulement, la comparaison est négative: «¡No soy un tractor ni un carro!» (30), mais cette occurrence ne fait que confirmer la règle selon laquelle le corps est perçu comme déshumanisé, comme mécanisé. Une autre occurrence établit le lien entre corps et machine de manière particulièrement forte puisqu'il y a une sorte de va-et-vient entre comparé et comparant: «Y el corazón como un motor fallando, a punto de pararse, de eyacular» (62). Dans un premier temps, le cœur, le comparé, élément humain est assimilé à un moteur, le comparant, puis le narrateur attribue à ce moteur, qui devient donc à son tour, un comparé, une action humaine ou tout au moins animale, «eyacular», le corps étant alors le comparant. Le statut de comparé et celui de comparant semblent ainsi s'échanger, ou plutôt s'annuler, ce qui a pour effet, une sorte de fusion ontologique entre corps et machine. Leur Être est de même nature.

Cette phrase nominale est véritablement au cœur du paradigme corpsmachine: en effet, elle réunit les deux thématiques toujours en jeu dans les parallèles homme / machine que Vallejo propose: celle de la sexualité et de la reproduction, et celle du dysfonctionnement. La première intervient dès les premières pages du récit: «el matrimonio perfecto. Nueve hijos fabricaron en los primeros viente años mientras les funcionó la máquina» (8, nous soulignons) $)^{3}$. Remarquons que le dysfonctionnement est déjà présent lui aussi, il est du reste plus présent tout au long du récit, ce qui est parfaitement logique: le corps étant peu érotique, le discours concernant la sexualité est assez réduit, en revanche, la faillite du corps étant omniprésente, le discours sur ses dysfonctionnements est beaucoup plus nourrit. L'un des exemples les plus éloquents est celui-ci:

Pensé que te había vuelto a descomponer el teléfono.

No, el descompuesto era él que se estaba muriendo (11).

3 Voir aussi p. 59: «la máquina reproductora».

4 Voir p. 64, «Esta mujer que parecía zafada, tacada del coconut como si tuviera el cerebro más desajustado que los tobillos»; p. 16, «Y le hacían cortocircuito». Nous soulignons. 
Encore une fois corps et machine sont interchangeables dans leur faillibilité. Ces divers propos sur le corps sont marqués par un pessimisme exacerbé. Toutefois, Vallejo semble construire une sorte de justification - et n'allons pas jusqu'à dire argumentation - autour de sa vision en se référant à des discours de type scientifique ou bien philosophique.

La conception du corps comme machine n'est évidemment pas nouvelle, et Vallejo en a parfaitement conscience puisqu'il fait une référence discrète à Descartes dès l'ouverture de son récit. Descartes est le philosophe qui a le plus contribué à la diffusion de cette théorie des animaux-machines, dans la cinquième partie de son Discours de la Méthode, notamment. El desbarrancadero en décrivant un oiseau métallique: «Un pájaro cortó el aire seco con un llamado inarmónico, metálico» (12) crée un intertexte avec Descartes. Les deux adjectifs font immédiatement penser à un son émis par une machine. Souvenons-nous que Descartes prenait pour exemple de ces animaux-machines, semblables à des "automates», un perroquet (Descartes 1953: 164-165).

La pensée de Descartes n'est pas la seule à figurer dans le récit. Vallejo reprend une très ancienne métaphore du corps (encore une fois, un corps malade), celle du corps politique ${ }^{5}$ :

¿Qué va, Colombia no se acaba! Hoy, la vemos roída por la roña del leguleyismo, carcomida por el cáncer del clientelismo, consumida por la hambruna del conservatismo, del liberalismo, del catolicismo, moribunda, postrada, y mañana se levanta de su lecho de agonía, se zampa un aguardiente y como si tal, déle otra vez, ¡al desenfreno, al matadero, al aquelarre! (84-85, nous soulignons)

On le voit, la Colombie est un pays malade: un corps dévoré par le cancer, comme le père du narrateur; la patrie (qui n'en est plus vraiment une ${ }^{6}$ ) se superpose donc au père dans une sorte de longue agonie par un double jeu de langage, métaphorique et étymologique. La relation au père, comme à la patrie, est marquée du sceau de la mort: c'est le narrateur qui met fin aux souffrances de son père, et ce même narrateur définit toujours son pays comme celui de la mort et des assassins. Le pays souffre de ses hommes politiques, les récits de Fernando Vallejo le répètent inlassablement. Le

5 Il est hors de question de refaire ici toute la généalogie de cette métaphore qui a connu un vif succès à toutes les époques: Platon, Hobbes (dès l'introduction de son Léviathan), Rousseau dans le Contrat Social, pour ne citer que quelques exemples.

6 Voir p. 8: «¡Cuál Dios, cuál patria! ¡Pendejos! Dios no existe y si existe es un cerdo y Colombia un matadero.» Il y aurait évidemment beaucoup à dire sur les rapports passionnels que l'auteur et le narrateur entretiennent avec la Colombie. 
corps en vient presque à acquérir dans ce texte une sémantique davantage liée à la déchéance qu'à la vie proprement dite. Le terme "corps » devient un comparant non plus pour prédiquer le sème / quelque chose de vivant / mais celui de/ quelque chose de mourant/.

Dansl'exemple précédent, Vallejo s'inscrit dans une filiation métaphorique claire, mais il va plus loin, la métaphore est également réactivée, renouvelée, dans un autre passage: "Pero el país funciona muy bien. Con mordida todo fluye: el tráfico de los carros, la venta de electrodomésticos, la circulación de la sangre, las putas del presidente, los pasaportes de los que viajan, los entierros de los que se van... La mordida es un invento genial. Como la rueda» (138). La présence de l'évocation du sang dénote au milieu de l'énumération: cette fois-ci nous n'avons plus affaire au corps politique mais à la politique du corps, ou qui gère le corps, dans une sorte de renversement reflétant un pays qui "marche sur la tête».

Autre filiation omniprésente, celle de la danse macabre et du memento mori. Nous avons déjà évoqué la personnification de la syphilis en danseuse lascive, mais ce n'est qu'un élément qui entre dans le cadre de la danse macabre. La mort est traitée comme un personnage à part entière, elle apparaît à plusieurs reprises, souvent souriante $(10,29 \ldots)$ et le narrateur lui parle (49-50). Les personnages de mourants ${ }^{7}$ - en particulier, Darío, qui est décrit comme "un cadáver de treinta [kilos]» (24) bien qu'il soit encore en vie - viennent compléter la ronde, ainsi que tous les Colombiens : «Colombia se dividía en conservadores y liberales. Hoy se divide en asesinos y cadáveres» (130). C'est tout le pays qui est assimilé à un vaste cimetière. L'incertitude entre mort et vie revient à plusieurs reprises («revivir cadáveres », 28; "por fin acabó de matar al cadáver», 77); nous avons déjà évoqué plus haut les "zombies», donc des morts-vivants, qui ont remplacé les «muchachos de Junín» pleins de santé.

À la danse macabre, plutôt d'origine médiévale, s'ajoutent le memento mori et le tableau de vanité qui connurent un grand succès à l'époque baroque. L'évocation du corps en plein pourrissement et le festin des "gusanos » (21, $66,83,93$ ) ou bien en l'état de squelette ("polvo», 22; "cadáver», 22, 24, 28,137 ; «los huesos », 21, 41) est récurrente. Vallejo renoue avec l'esthétique du macabre, mais le message moral qui l'accompagnait dans les siècles passés a disparu: il n'est pas question de résurrection chez Vallejo, la mort n'est

7 Évoquons à ce sujet le personnage de El Difunto dans La Virgen de los sicarios (44, 47), ayant miraculeusement survécu à une exécution, se réveillant au milieu de son enterrement, qui restera après sa sortie de cercueil une sorte de mort-vivant. 
pas un passage mais bien une fin. Le pourrissement du corps vaut pour luimême, peut avoir de l'intérêt car il est la seule certitude avant le néant total.

Le propos de Vallejo concernant le corps s'appuie également sur des discours disciplinaires plus spécifiquement scientifiques. Comme on peut s'en douter, le discours médical est omniprésent, pas question donc de faire un relevé exhaustif des occurrences. On peut en revanche discerner quelques caractéristiques propres au discours médical ou sur la médecine de Vallejo.

Il y a tout d'abord ce que l'on pourrait appeler le discours du narrateur à coloration médicale. À plusieurs reprises le narrateur, devant la détresse de son frère - il est régulièrement question de la dignité du malade, se substitue aux médecins; en lui administrant des traitements "alternatifs»: un anti-diarrhéique pour les bovins (les traitements humains n'étant plus efficaces, 15), en lui conseillant de fumer de la marihuana pour lui redonner de l'appétit (16). Il donne aussi son avis sur les maladies et les symptômes, avis plus ou moins fondé et recevable d'un point de vue scientifique (15, $21,25,42,62,77 \ldots)$. Les véritables médecins, eux, sont en général assez maltraités par le narrateur "yo odio a los médicos» (60) et le lui rendent bien ( «A mí los médicos me detestan, no sé por qué. Tal vez porque les hago pasar examen y les quiero hacer revalidar el título » 26); ils sont considérés comme des incompétents, à la limite assimilés à des croque-morts: «Para eso [bien morir] han estado siempre los médicos, para desbarrancarnos, con la bendición del cura, en el despeñadero de la eternidad» (93-94). D’autres éléments concourent à cette parodie ou cette satire de la médecine: une scène notamment où il est question de protocoles médicaux.

Que la curación de un paciente no pasaba de ser "un caso anecdótico", que eso no era ciencia. Ciencia era, para empezar, mil pacientes cuando menos con diarrea y sida enrolados en un protocolo de "double blind" o doble ciego.

¡Para doble ciego yo, doctor, que tengo las dos córneas trasplantadas de sicarios y veo por todas partes policías y alucino con qué mato médicos! (26)

La parodie, sur ton de délire, est quand même assez féroce et grinçante. Même lors de la scène où le narrateur se remémore l'euthanasie du père par injection létale, l'ironie est présente. Le récit passe à la troisième personne créant un dédoublement du narrateur, entre celui du passé, qui correspond à la troisième personne, et celui qui raconte, à la première personne (phénomène qui apparaît déjà dans La Virgen de los sicarios) ${ }^{8}$.

8 Le phénomène apparaît dans les deux récits dans des moments où l'émotion est intense, comme si le narrateur avait besoin, par le passage à la troisième personne, de mettre de la distance entre lui et la situation insoutenable. 
Tomó el Eutanal. ¿Y saben qué hizo? Con algodón que empapó en alcohol desinfectó el tapón de caucho del frasco. ¡Como si el Eutanal fuera un remedio! ¡Y como si los muertos se pudieran infectar! (131-132)

Ici l'ironie frappe cruellement le personnage, mais aussi la médecine en faisant d'un geste prophylactique un geste automatique, dénué de sens. C'est donc la médecine elle-même qui devient, à son tour, comme le corps, mécanique. Vallejo en faisant la satire de la médecine s'inscrit une fois encore dans une longue tradition, celle de Molière, par exemple.

Mais Vallejo n'est pas toujours ironique avec les discours scientifiques auxquels il se réfère, lorsqu'il fait allusion à la physique, à la mécanique. Toutes ces allusions viennent en effet étayer la démonstration de la thèse qui est contenue dans le titre de son récit: les corps / les vivants sont faits pour chuter dans le précipice de la mort. Le texte présente une isotopie extrêmement nourrie autour de l'idée de chute, de vide9. C'est la physique newtonienne qui vient expliquer cette obsession. Sur un mode direct ou sur un mode métaphorique, de nombreux éléments en rapport avec la physique et plus particulièrement la mécanique apparaissent au fil du récit: «la fuerza de la gravedad» (14), les orbites de rotation des corps (20), «electrón» et «átomo» (147), apparaissent aussi les différentes lois de la physique: «ley de la gravedad» (166), du chaos (21), celles de Murphy $\left(69,125\right.$ et 106 $\left.{ }^{10}\right)$, mais également les physiciens eux-mêmes: Einstein $(140,146,161)$ et Newton (69-70). Les lois de la gravité, formulées par Newton, sont détournées pour expliquer la fascination du vide (conduisant à la mort) que ressentent les êtres humains et particulièrement les personnages du récit. Un corps physique est soumis à la gravité, donc un corps humain tend à tomber dans le «desbarrancadero de la muerte» (7). Vallejo réécrit la loi de gravité universelle à partir du double sens de «cuerpo». On voit bien que le corps est central dans le récit, en tant que thème, mais aussi en tant que mot: c'est le noud de la narration.

Ce qu'il fait pour Newton, il le fait aussi pour Einstein:

El que vive mucho carga con muchos muertos, es natural. Así lo establece la primera ley de los vivos o ley de la proporcionalidad de los muertos, que yo descubrí y que estipula una relación directa entre los años que vive el cristiano y los muertos que carga, cargando más el que vive más: $\mathrm{v}=\mathrm{m}^{2} \mathrm{~d}$

9 Relevé indicatif: en rapport avec la chute $(7,61,63,74,159,169,182,192$, ce dernier exemple rappelant la Chute originelle); avec le précipice $(7,74,94)$; «abismo» (156); en rapport avec le vide ou le trou sans fond $(92,98,99,101,187)$.

10 Déjà évoquées dans La Virgen de los sicarios (98). 
(uve igual a eme al cuadrado por de), donde $\mathrm{v}$ es vivo, $\mathrm{m}$ es muerto y d la constante universal del desastre, que por ser una «constante» cambia « constantemente» como el espacio de Einstein: se curva, se encoge, se estira, se expande, se alarga. (140)

La parodie de $\mathrm{E}=\mathrm{mc}^{2}$ vient illustrer le pessimisme de Vallejo et le poids que peut avoir la mort sur la vie. Quant aux lois de Murphy qui précisent que si une situation a la possibilité d'empirer, elle empirera forcément à un moment ou un autre, Vallejo la fait évidemment sienne sans la modifier véritablement, en ajoutant à peine un clin d'œil à Candide et à Leibniz: «por las leyes de Murphy que rigen el Universo, todo en el peor de los mundos tenía que andar mal» (125). Les lois de la physique sont donc convoquées pour «justifier» le pessimisme de Vallejo et son obsession de la finitude.

Vallejo a donc inscrit son récit dans une série de filiations, qui ne sont plus forcément très actuelles, se plaçant ainsi à contre-courant de son époque. Le pessimisme général du narrateur rappelle la théorie heideggerienne du Sein zum Tode (l'être-pour-la-mort) formulée dans Sein und Zeit: l'être humain a pour finalité la mort. Vallejo avec sa métaphore du desbarrancadero ne dit pas autre chose, la seule certitude humaine est celle de la mort, mieux, il en fait une sorte de synthèse puisque sa métaphore fait aussi allusion à la notion de «fond-sans-fond» présente dans la pensée d'Heidegger; ajoutons à cela que l'expression «leer a Heidegger» apparait à deux reprises, dont une fois au centre du récit (106 et 127). Ce récit peut donc se lire comme une mise en œuvre ou une démonstration de la philosophie d'Heidegger que Vallejo fait converger avec les lois de la physique. Les deux discours se superposent pour venir justifier la pensée pessimiste de Vallejo. Ainsi le corps met d'accord les deux disciplines, il sert de carrefour pour la rencontre de la science moderne et de la philosophie.

\section{Bibliographie}

Descartes, René, 1953, Discours de la méthode, in Euvres et lettres, Paris, Gallimard, Bibliothèque de la Pléiade.

Semilla Durán, Marián, 2004, "A la búsqueda del “irrecuperable fantasma": autobiografía, autoinvención y ficción en El río del tiempo de Fernando Vallejo» in Maryse Renaud (éd.), Espejismos autobiográficos, Poitiers, CRLA, p. 377-390.

Vallejo, Fernando, 2001, El desbarrancadero, Madrid, Alfaguara.

Vallejo, Fernando, 2006, La Virgen de los sicarios, Madrid, Punto de lectura; (1e édition: 1994). 